\title{
Artificial Life and Natural Intelligence
}

\author{
Keith L. Downing \\ The Norwegian University of Science and Technology \\ Trondheim, Norway \\ keithd@idi.ntnu.no
}

\begin{abstract}
This paper reviews the neuroscience literature to sculpt a view of intelligence from the artificial life (ALife) perspective. Three key themes are used to motivate a journey down the low road to cognition. First, the origins of brain structures and dynamics exhibit considerable emergence at phylogenic, epigenetic, and ontogenetic levels. Second, ALife complexity measures have interesting parallels in theoretical neuroscience. Finally, the cerebral internalization of sensory stimuli and motor control explain, respectively, a) semantics in terms of differential complexity, and b) how neural evolution has overcome the limitations of simple emergence.
\end{abstract}

\section{Introduction}

The vast majority of Artificial Life (ALife) research involves large populations of extremely simple components whose collective behavior yields emergent sophistication. Often, these local units mimic simple biological organisms such as bacteria, or serve as very high-level abstractions of complex organisms - e.g., simulated economic agents whose intelligence is restricted to a few simple buying and selling activities.

The realm of high-level intelligence is traditionally unpopular in ALife for several reasons. First, intelligence issues are often philosophical quagmires where unwary visitors may disappear without a trace (of publishable work). Second, the field of Artificial Intelligence (AI) once boldly marched into that dark swamp, spouting claims of human-like computers just around the corner, thus fueling rampant media hype. AI only barely escaped (from both the swamp and the media) and has now retooled considerably to focus on intelligent systems without significant anthropomorphic claims. ALife researchers are wary of a similar fate, via either intelligence work of their own or merely association with AI. Finally, the concept of complex localized controllers (i.e., brains) runs contrary to the ALife philosophy of emergent global regulation from simple components.

Although intelligence is truly one of life's most perplexing riddles, contemporary neuroscience has made incredible strides in the past few decades, thus burying many stale theories of mind that survived far too long due to lack of reasonable evidence. In a very strong sense, the neurophysiological evidence yields intelligence even more foreboding than before. Now that we understand many of 
the local mechanisms such as neurotransmitter signaling, synaptic strengthening, neuronal migration and axonal growth, the distance from these primitives to cognition seems all the more ominous. The old theories of a CPU-like humunculous that ran the show were much easier to work with, but the von Neumann computer analogy was wrong, and, unfortunately, very misleading.

The relevance of intelligence for ALife is now obvious. Since no sophisticated central controller runs the brain, intelligence itself emerges from the interactions of billions of neurons distributed across many brain regions. Cognitive processes such as prediction, memory, learning, etc. should thus, ultimately, find explanations in the cooperative and competitive interactions among neural agents.

This paper examines neurophysiological evidence and theories in terms of two common ALife concepts, emergence and complexity, before showing how natural selection of brains combats the limitations of purely emergent behaviors at one level through hierarchical organization and ascending control.

\section{Emergence, Adaptation and Intelligence}

Understanding intelligence from an emergent perspective involves both a) selforganization of structural and dynamic patterns within a given organism, and b) the crafting of sophisticated life forms through natural selection. Both mechanisms underlie life's 3 key adaptive mechanisms: a) development, b) learning, and c) evolution.

This paper reviews several basic mechanisms that are helpful in understanding the phylogenic (evolutionary), ontogenetic (developmental) and epigenetic (learning) aspects of emergent intelligence, and thus useful in tackling questions such as:

1. How can brains capable of intelligent behavior evolve?

2. How are neural topologies grown from genetic instructions?

3. How do neurons interact to facilitate intelligence?

\subsection{Basic Mechanisms for Emergent Intelligence}

Duplication and Differentiation Something as complex as the brain cannot emerge in one evolutionary step, although this does not preclude a mixture of punctuated and gradual refinements over the millennia. Each incremental change, whether positive, negative or neutral with respect to intelligence, cannot compromise the species overall fitness. One simple means of guaranteeing a relatively monotonic progression is to duplicate existing genes and then allow the copies to gradually mutate until they achieve an intelligence-enhancing variant. While the copy explores function space, the copied gene continues to perform its normal role, thus providing protective fitness cover during exploration.

The classic illustration of this mechanism is the homeobox, a 180-base-pair DNA sequence found in the homeotic genes. Similar sequences of homeotic genes appear in organisms as simple as hydra and fruit flies and as complex as 
chickens and humans [1]. The homeobox (along with other peripheral base pairs) has clearly been duplicated many times throughout evolution, with peripheral regions then differentiating to form more heterogeneous phenotypes. Incidentally, several of the homeotic genes are involved in the development of the hindbrain, the most primitive cerebral region.

During development, cellular duplication and differentiation are critical activities. After fertilization, the zygote undergoes rapid cleavage divisions to form the blastula, consisting of many identical copies of the original cell. Small asymmetries in the blastula eventually lead to differential gene expression among the cells, causing differences in inter-cellular chemical signaling, leading to further differentiation and an ensuing escalation in complexity.

The blastula transforms into the 3-layered gastrula, Within its neuroectoderm, all cells have the potential to become neuroblasts (neuron precursors). Random asymmetries lead to the formation of isolated neuroblasts, which then send chemical signals that inhibit nearby neuroblast formation and promote epidermal cells.

Migration and Extension Neuroblasts migrate to the center of the gastrula to form the ventricular proliferation zone (VPZ), where they differentiate into either neurons or glial cells. Neurons undergo further differentiation to their final neural cell type before migrating back outward along the radial glial scaffolding. The brain thus forms from the inside out, as neurons migrate past their temporal predecessors to the periphery.

Since developmental timing effects in the VPZ can strongly influence eventual neural cell fates, a few simple (often genetically-controlled) timing effects can greatly alter the final brain anatomy in terms of the number and thickness of neural layers. Nonlinear competitive and cooperative interactions between the neurons of these layers can then lead to vastly different connection topologies as well. Essentially, neural development is a process poised on the edge of chaos, where small changes to initial (timing) conditions can have large-scale repercussions.

Once properly positioned, neurons sprout axonal and dendritic projections, with the former often growing many centimeters in length. The vast complexity of brain function stems largely from the ability of neurons to send direct, nondiffuse, signals to other particular (often distant) neurons. In contrast, a network in which neurons could merely broadcast signals within variable-sized radial neighborhoods could probably never achieve the same level of sophistication as the vertebrate nervous system.

As described in [19], 4 primary factors control axonal navigation: chemical attractants and repellents that either diffuse within intercellular spaces or cling to cell surfaces. Axons wind their way through fields of diffused attractants and repellents, while bouncing off repellent-laden cells and growing along certain surface attractants. Chemical signatures in the two neurons appear to determine the targets to which growing axons become synaptically coupled. In general, chemical signals direct axons into proper brain regions and to sub-populations 
of compatible dendrites, but finer tuning of interneural connections involves competitive and cooperative interactions based on correlated firing patterns.

Cooperation In 1949, Donald Hebb [6] predicted that:

When an axon of cell A is near enough to excite a cell B and repeatedly or persistently takes part in firing it, some growth process or metabolic change takes place in one or both cells, such that A's efficiency as one of the cells firing $\mathrm{B}$, is increased.

In short, neurons that fire together, wire together. Neurophysiological research has revealed the underlying metabolic changes and largely confirmed Hebb's hypothesis [9]. This mechanism is central to both biological learning and associative memory formation in various artificial neural networks [7].

Hebb's rule embodies cooperation in at least two respects. First, and most obviously, by (nearly) simultaneously firing, neurons A and B work together to strengthen their intervening synapse. Second, and often overlooked in Hebb's quotation, is the clear implication that $\mathrm{A}$ is one of several neurons that actually stimulate B. This cooperation among presynaptic neurons underlies classical conditioning, wherein an initial C-B association essentially bootstraps an A-B association.

For example, assume a basketball player normally dribbles to the right (DR) when he sees the nearest defender moving to (the defender's) right (MR). With experience, he will notice that a defender quickly shifts his body weight to the left leg (SL) before moving to his right (MR). Eventually, this will lead to a hard-wired response wherein SL initiates DR: the dribbler moves right on seeing the weight shift and without waiting for MR.

As simplified in Figure 1, assume 3 neurons (more likely, 3 possibly-overlapping populations of neurons) that represent DR, MR and SL, respectively. Here, DR is the post-synaptic neuron, while SL and MR are pre-synaptic (i.e. they send signals across separate synapses to DR). Bootstrapping embodies cooperation in the following sense. MR is normally sufficient to fire DR. In situations where MR and SL fire almost simultaneously, MR still fires DR, but now SL and DR are also almost simultaneous. Hence, the SL-DR synapse strengthens such that, later, SL alone eventually suffices to fire DR. Essentially, MR has primed DR to enable the SL-DR association.

Dropping down a level, coincidence detectors are essential prerequisites for neural cooperation. In classical conditioning, NMDA receptors in the post-synaptic neuron's dendrites recognize the simultaneity of a) depolarization (firing) of the post-synaptic neuron (e.g. DR), and b) neurotransmitter release by the axon of the pre-synaptic neuron (e.g., SL). Only when both occur does the NMDA receptor open its associated calcium channel, setting off a chain of events that eventually enhance the SL-DR synapse [9].

In the pre-synaptic axon, adenyl cyclase (AC) detects the co-occurrence of a) pre-synaptic neuronal firing, and b) a general signal of excitement in the form of a neuromodulator (e.g. serotonin) broadcast to many parts of the brain by 


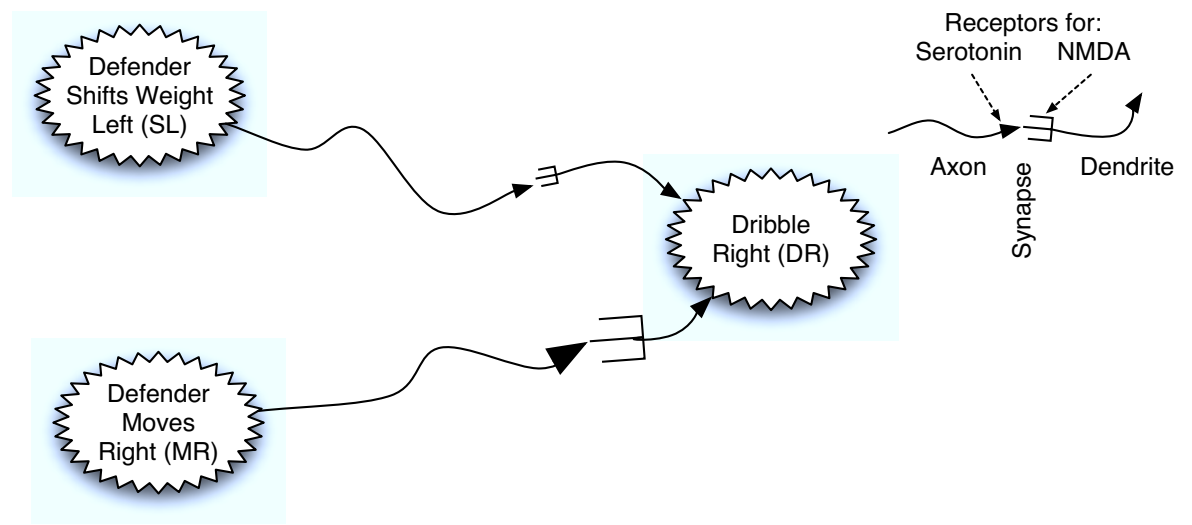

Fig. 1. Abstraction of neural circuitry involved in classical conditioning for learning to dribble right when the opponent shifts his body weight to his left side.

the limbic system during emotional arousal. This is a key mechanism behind operant conditioning, where proper actions are learned via punishment/reward. For example, as our basketball player is learning to shoot free throws, different sets of motor neurons correspond to particular shooting actions, since different sets trigger different muscle fibers. If a particular free-throw is good (due to the firings of the motor-neuron set $\mathrm{M}$ ), then the emotional experience of success leads to serotonin release (by broadcasting neurons in the limbic system). The $\mathrm{AC}$ in the pre-synaptic axons of the $\mathrm{M}$ neurons will then detect the coincidence of serotonin and recent depolarization, thus leading to prolonged neurotransmitter release (via a complex, but well-understood [3] reaction sequence) at those same M-neuron synapses in the future. In short, AC's co-detection of success/excitement and M-neuron activity enhances the cooperative firing of the M neurons in the future.

This is one of many areas where a proposed ALife primitive is subsumed by other ubiquitous mechanisms. However, since coincidence detection forces a descent to the chemical level, where complexity quickly escalates, this paper will remain at a higher level of abstraction. Also, the general success of artificial neural networks (most of which abstract away all neural physics and chemistry) indicates that lower levels are not necessarily essential for artificial intelligence.

Competition Whereas NMDA and AC underlie cooperation, chemical growth factors known as neurotrophins incite intense competition among neurons. Axons are dependent upon neurotrophins for growth and maintenance; without them, they wither away. During development, axons extend toward their targets, whose dendrites give off neurotrophins in inverse proportion to their activity. The limited supply of neurotrophins thus supports a restricted number of 
presynaptic axons, with the rest atrophying away. The proper match of axons to target dendrites (and thus a proper level of target activity) emerges from a simple negative-feedback loop: low target activity stimulates neurotrophin release, which positively affects presynaptic axonal growth, and more axons provide greater input to the target, increasing its activity.

Once the basic neural topology is established, neurotrophins also play an important role in learning, but via slightly different dynamics. In the mature brain, neurotrophins are released by depolarized dendrites and only taken up by recently-depolarized pre-synaptic axons. Hence, only those axons that contribute to the stimulation of the target neuron are rewarded with growth factor, while the others lose out in this competition for the fruits of cooperation.

Competition for neural growth factors may explain the formation of topological maps in the brain, i.e., regions that have an isomorphic relationship to some aspect of the environment. For example, neurons in layers 5 and 6 of the V1 area of the visual cortex respond maximally to lines at particular orientation angles in the visual field [8]. Most significantly, a) neighboring cells respond to similar angles, and b) horizontal transects represent a continuous sequence of monotonically changing preference angles. In short, the neuron space is isomorphic with orientation-angle space. A multitude of such maps exist in the brain, covering all types of sensory input. In fact, many of the initial levels of perceptual processing involve topological maps. Only in higher brain regions do the isomorphisms disappear, as sensory channels converge with one another and with top-down cognitive biases.

As detailed in [17], several different competition-based neural models suffice to generate topological maps of visual orientation angles, via self-organization. These include the classic Kohonen maps [11] in which post-synaptic neurons compete for pre-synaptic firing patterns. Our preliminary modelling efforts indicate that maps can also be generated by Kohonen-type networks based on a simple model of neurotrophin release and uptake.

\subsection{The Emergent Integrated Hierarchy of Intelligence}

The above primitives interact to form complex cognitive systems whose overall topology is convergent (i.e., high axonal feed-in from many regions), reentrant (i.e. loop-forming) and hierarchical (i.e., a series of layers whose functionalities vary along a spectrum from specific to general). For example, Figure 2 depicts some of the connections between sensory topological maps and the hippocampus, believed to be the center of long-term memory [9]. Many brain researchers agree that two key features of higher intelligences are convergence and reentrance/recurrence $[13,5]$.

Convergence enables the integration of perceptual inputs. This helps provide the holistic awareness of a situated and embodied self, which many consider the basis of consciousness $[4,5,14]$. Many researchers point to the thalamus as the center of consciousness, since it combines multi-modal sensory inputs with memories and then feeds back to the sensory areas of the cerebral cortex, thus forming a memory/contextual bias on further perception. Consciousness is then 


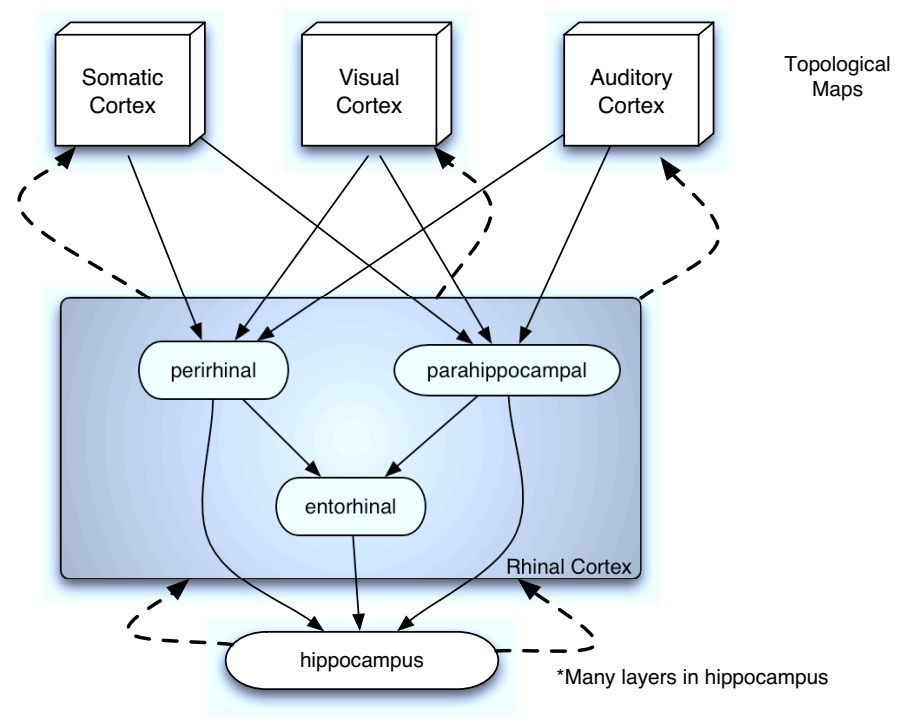

Fig. 2. Convergence and reentrance among the many hierarchical layers from the cortical maps to the hippocampus

more than just a simple awareness of present self and environment, but a partial integration of generalized-past and filtered-current experience.

Although convergence, recurrence and tight integration among components appear to be fundamental emergent properties of the brain, they are normally eschewed by engineers, who prefer systems of loosely-coupled, largely-independent, modules, since these are more amenable to top-down, divide-and-conquer design strategies. Hence, if considerable convergence and recurrence are necessary hallmarks of higher intelligence, then the design of truly artificial intelligences may actually require bottom-up selectionist techniques, such as evolving artificial neural networks.

In summary, the three adaptative mechanisms are essential prerequisites to higher intelligence, and each is grounded in (at least) the above basic mechanisms. Duplication and differentiation are necessary for genotypic and phenotypic complexification, since natural selection severely punishes most exploratory designs. They, along with migration and extension, are also fundamental tools of neural development. Also, beyond their obvious evolutionary influences, cooperation and competition are actively at work in development and learning.

Below this level lies a fascinating array of chemical devices, of which this paper only scratches the surface. Above it come emergent structures such as sensory maps, convergence zones and recurrent loops, whose integrated activity spawns abstract mental phenomena such as memory, awareness, decision-making and consciousness. 


\section{Complexity and Consciousness}

Concepts such as intelligence and consciousness have successfully avoided precise formalization for centuries. However, Edelman and Tononi [5, 18] provide a nice break from the philosophical confusion via a statistical quantification of neural complexity - and one which closely parallels classic ALife notions such as the edge of chaos [10,12] and self-organized criticality [2]. Also see [16].

The basic idea is quite simple: complex neural systems are those in which different regions can be in a large number of different states, but these states are highly correlated with the states of other regions. This motivates the expression: differences that make a difference, i.e., the (many) states of particular regions strongly influence the states of others.

Edelman and Tononi's complexity involves mutual information (MI), which is based on the comparative entropies $(\mathrm{H})$ of two subsystems (A and $\mathrm{B}$ ) and their union:

$$
M I(A, B)=H(A)+H(B)-H(A \cup B)
$$

High entropy systems are those with many equiprobable states, so high mutual information involves subsystems with many equiprobable states but with few such states in the union, i.e. low $H(A \cup B)$, which directly reflects a high correlation between A and B.

Then, the neural complexity $(\mathrm{CN})$ of an n-neuron system $(\mathrm{S})$ involves the mutual information between every subsystem $\left(s_{j}\right)$ and its complement $\left(S-s_{j}\right)$, summing over the average MI values for each size class $(\mathrm{k})$ :

$$
C N(S)=\sum_{k=1}^{n / 2} \overline{M I\left(s_{j}^{k}, S-s_{j}^{k}\right)}
$$

A complex neural system is therefore one containing many subsystems that are both internally diverse and mutually integrated. Levels of diversity and integration show characteristic variations according to brain maturity [5], as shown in Figure 3.

1. Young brains exhibit high integration but low diversity, producing activity patterns in which many neurons in $\mathrm{S}$ synchronously change state, but local regions show few activation patterns. Hence, the system appears to run through low-period cycles of highly homogeneous states.

2. Old brains are weakly integrated but highly differentiated, leading to chaotic activation patterns in which each region behaves independently.

3. Mature brains portray both high integration and differentiation, thus producing long state cycles where each state has a structured (low entropy) appearance, but these patterns gradually change in a continuous manner, as a glider moves across a cellular-automata. They look alive!

In terms of the neural complexity metric:

$$
C N(\text { old })<C N(\text { young })<C N(\text { mature })
$$




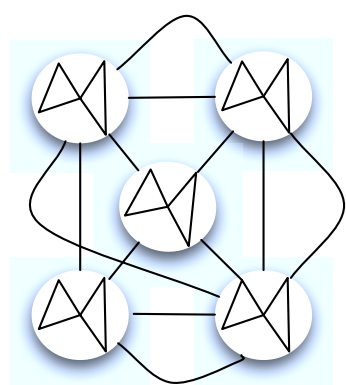

Young

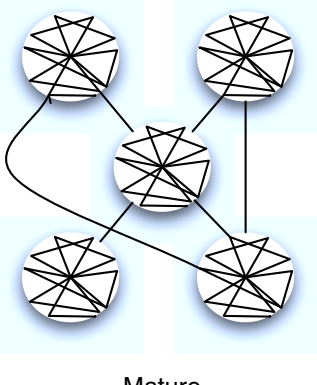

Mature

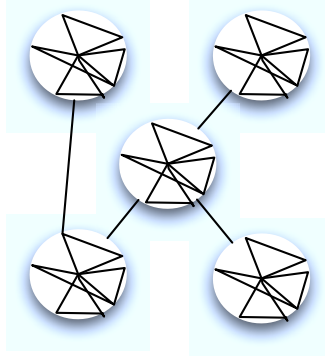

Old

Fig. 3. Abstraction of neural topologies in young, mature and old brains, showing degree of connectivity both within and between different regions.

The parallels to ALife complexity definitions are striking. Old brains mirror a gaseous state, where chaos dominates. Young brains resemble a solid state, where state cycles are short. However, in contrast to a Kaufmann solid [10], where large regions are static, young brain cells do frequently change state, but individual regions show only a few local patterns: they are frozen into a small set of alternatives. Finally, the mature brain closely matches the liquid state, the edge of chaos, and self-organized criticality in that it has long state cycles and appears to exhibit both memory and information transfer as patterns gradually form and retain much of their shape as they move about.

Neural complexity links to consciousness via a few additional concepts [5]. First, a functional cluster is a neural region with a higher cluster index than any of its subset regions, where the cluster index (CI) is the ratio of a regions internal to external interactivity:

$$
C I\left(S_{i}\right)=\frac{\sum_{j=1}^{m} H\left(s_{i, j}\right)-H\left(S_{i}\right)}{M I\left(S_{i}, S-S_{i}\right)}
$$

Here, the numerator denotes the integration $\left(I\left(S_{i}\right)\right)$ of subsystem $S_{i}$ : the amount of entropy that $S_{i}$ 's m subsystems $\left(s_{i, j}\right)$ lose due to their mutual interactions. The denominator reflects $S_{i}$ 's contribution to S's complexity.

A functional cluster whose interface with its complement $\left(S-S_{i}\right)$ may change on approximately a $1 / 10$ second timescale is a dynamic core [5]. It is hypothesized that the neurons involved in consciousness at a particular time are members of a dynamic core that involves extensive reentrant looping between the cerebral cortex and thalamus, and has high neural complexity, i.e., many different states that make a difference outside the core.

Thus, consciousness is a process governed by the ever-changing neural constituency of a thalamocortically-centered dynamic core. As in ALife systems, a) the dynamics, not the substrate, define the phenomena, and b) the emerg- 
ing global pattern exerts a strong influence upon the system, e.g., by biasing perceptual interpretations, the focus of attention, memory retrieval, etc.

\section{Encephalization}

Traditional ALife complexity analyses concern the effects of external perturbations upon internal dynamics, where the disturbance is small - such as the addition of one sand grain to a pile or the flipping of one bit in a quiesced boolean network - and the probability distribution over the sizes of the effects differentiates between the stable, chaotic and complex regimes.

However, living organisms interact with a complex environment whose perturbations are both intricate and extensive; and the resulting imprint on internal dynamics constitutes an adaptive response with real survival value. The view of brain and surroundings as coupled autopoietic (i.e. constantly self-maintaining) systems [15] captures this idea:

The plastic splendor of the nervous system does not lie in its production of engrams or representations of things in the world; rather it lies in its continuous transformation in line with transformations of the environment ... The functioning organism, including its nervous system, selects the structural changes that permit it to continue operating, or it disintegrates (pg. 170).

Basically, salient aspects of the environment are internalized in the neural circuitry, or encephalized. To quantify this effect, Tononi et. al. [18] use CN to define matching complexity, CM: the complexity of the system due to sensory input, or, the degree to which internal correlations change due to external perturbations.

$$
C M(S, P)=C N(S)-C N(S-P)-C N^{E}(S, P)
$$

The matching complexity between a neural system $\mathrm{S}$ and its outer perceptual layer, $\mathrm{P}$, is the total complexity of $\mathrm{S}$ reduced by both the complexity of $\mathrm{S}-\mathrm{P}$ (the intrinsic complexity) and the complexity at the interface between $\mathrm{P}$ and $\mathrm{S}-\mathrm{P}$ (the extrinsic complexity, $\left.C N^{E}(S, P)\right)$. Thus, CM measures the change in internal complexity due to the cascading effects of sensory input. This correlates well with stimulus familiarity, as seen in the experiments of [18], where ANNs trained on sample patterns and then exposed to similar new patterns show $C M>0$, while novel test patterns yield $C M<0$. Apparently, the familiar pattern calls a host of contextual information into play, producing much greater internal change than does a novel stimulus. Although a novel perturbation might cause great change to early levels of processing, its failure to link to previous experience quickly arrests any signaling cascades. Since CM seems to correlate with this memory/contextual factor, it implicitly measures the significance/meaning of a stimulus for the observer. Thus, the central ALife concept of complexity may provide insights into the philosophical conundrum of semantics. 
In addition to sensory inputs, encephalization can also encompass motor outputs, as detailed by Llinas [14]. Here, motor activity patterns that were originally emergent from the direct electrical couplings between muscle cells have, through the course of evolution, become controlled by, first, spinal motor neurons, and later, neurons of higher brain regions. This higher-level control increases the potential complexity of the actions, since emergent oscillatory patterns - typically, spatial waves of muscle contractions - cannot approach the intricacy needed for walking a balance beam or playing the piano. But with a neural hierarchy, spatial activation waves at the higher levels can, via tangled top-down connections, differential propagation delays, etc., cause spatially diverse firing patterns at lower levels.

Llinas [14] believes that muscle oscillations in primitive animals and in developing vertebrate embryos have become internalized/encephalized to the $40 \mathrm{~Hz}$ activity of the thalamus. Since thalamic activity is a critical constituent of the dynamic core, these $40 \mathrm{~Hz}$ oscillations serve as a binding signal for mental activity. In a strong sense, this $40 \mathrm{~Hz}$ signal is the heartbeat of the brain, and it arose via an evolutionary process that gradually translated emergent muscle-activation patterns into a high-level dynamic that coordinates all activity: perceptual, motor and cognitive. Thought is encephalized motricity.

Basically, evolutionary emergence combatted the limited motor complexity provided by simple emergence among locally-connected activators (i.e., muscle cells) by designing the nervous system, which permits intricate communication networks among non-adjacent cells. While primitive versions involve direct connections between sensory and motor apparatus, the brains of more intelligent organisms house many convergent and reentrant layers to realize high neural (and hence behavioral) complexity. This hierarchy manifests an encephalization of both environment and action, thus embedding reality for a selective survival advantage.

\section{Conclusion}

The potential synergies between ALife and neuroscience are abundant, albeit nonobvious. For instance, although a central controller is anathema to sciences of emergence, it becomes a crown jewel when its self-organizing processes are unveiled, as is now the case with many brain regions.

This paper has discussed several general mechanisms residing at an intermediate conceptual level between the electrochemical and the psychological. In all cases, these principles are quite well understood in terms of the lower levels, so they provide well-grounded intellectual scaffolding for bottom-up attacks on cognitive phenomena such as learning and memory.

Ideally, ALife systems could begin with these processes and derive intelligence. AI was driven by similar optimism, but primitives such as logic and bestfirst search had no obvious neural basis. Conversely, the staples of ALife (competition, cooperation, differentiation, etc.) do. In addition, ALife-related concepts 
such as neural complexity $(\mathrm{CN})$ and matching complexity $(\mathrm{CM})$ provide elegant metrics for cognitive emergence.

In general, the study of intelligence in terms of neural networks gives ALife researchers a host of interesting opportunities: 1) to understand complex phenomena such as memory, reasoning and semantics from an emergent perspective, 2) to recognize and formalize the limitations of behavioral emergence and the improvements accrued by a nervous system, 3) to quantitatively analyze the informational coupling between environments and brains as they coevolve, and 4) to analyze similar informational correlations between motor patterns and their proposed encephalizations in controlling neural circuitry.

\section{References}

1. J. Allman, Evolving Brains, W.H. Freeman and Company, New York, NY, 1999.

2. P. BAK, C. TANG, AND K. WiESEnfEld, Self-organized criticality, Physica Review A, 38 (1988).

3. T. J. CAREw, Behavioral Neurobiology: The Cellular Organization of Natural Behavior, Sinaur Associates, Sunderland, MA, 2000.

4. P. Churchland, The Engine of Reason, the Seat of the Soul, The MIT Press, Cambridge, MA, 1999.

5. G. Edelman And G. Tononi, A Universe of Consciousness, Basic Books, New York, NY, 2000.

6. D. HeBB, The Organization of Behavior, John Wiley and Sons, New York, NY, 1949.

7. J. HopfiEld, Neural networks and physical systems with emergent collective computational abilities, Proceedings of the National Academy of Sciences, 79 (1982), pp. 2554-2558.

8. D. Hubel, Eye, Brain, and Vision, Scientific American Library, New York, NY, 1995.

9. E. Kandel, J. Schwartz, And T. Jessell, Principles of Neural Science, McGraw-Hill, New York, NY, 2000.

10. S. Kauffman, The Origins of Order, Oxford University Press, New York, 1993.

11. T. Kohonen, Self-Organizing Maps, Springer, Berlin, 2001.

12. C. Langton, Studying artificial life with cellular automata, Physica D, 22 (1986), pp. 120-149.

13. J. LeDoux, Synaptic Self: How Our Brains Become Who We Are, Penguin Books, Middlesex, England, 2002.

14. R. R. Llinas, $i$ of the vortex, The MIT Press, Cambridge, MA, 2001.

15. H. Maturana and F. Varela, The Tree of Knowledge: The Biological Roots of Human Understanding, Shambala Publishers, Boston, MA, 1998.

16. C. Nehaniv AND J. Rhodes, The evolution and understanding of hierarchical complexity in biology from an algebraic perspective, Artificial Life, 6 (2000), pp. 4567.

17. N. Swindale, The development of topography in the visual cortex: A review of models, Network: Computation in Neural Systems, 7 (1996), pp. 161-247.

18. G. Tononi, O. Sporns, AND G. Edelman, A complexity measure for selective matching of signals by the brain, Proceedings National Academy of Sciences, 93 (1996), pp. 3422-3427.

19. L. WOLPERT, Principles of Development, Oxford University Press, New York, 2002. 